\title{
TRANSVERSAL GROUP ACTIONS ON CR MANIFOLDS AND EMBEDDABILITY
}

\author{
ZoltÁn M. BALOGH AND CHRISTOPH LEUENBERGER
}

(Received October 30, 2001)

\begin{abstract}
We give necessary and sufficient conditions for embeddability of CR manifolds in terms of transversal group actions. These actions do not necessarily preserve the CR structure but they posses a holomorphic extension.
\end{abstract}

1. Introduction. A smooth manifold $M$ of real dimension $2 n+d$ is called a CauchyRiemann (CR) manifold if an $n$-dimensional complex subbundle $H^{1,0} M$ of $T^{C} M=C \otimes T M$ is specified that satisfies

$$
H^{1,0} M \cap H^{0,1} M=\{0\} \quad \text { and } \quad\left[H^{1,0} M, H^{1,0} M\right] \subseteq H^{1,0} M,
$$

where $H^{0,1} M=\bar{H}^{1,0} M$. We call $d$ the codimension of $M$ and if $d=1$ we say that $M$ is of hypersurface type. The bundle $H M$ is given as the real part by

$$
H M=\operatorname{Re}\left(H^{1,0} M \oplus H^{0,1} M\right) .
$$

Since we focus on local questions in this paper it can be assumed that $M$ is an open neighborhood of the origin 0 in $\boldsymbol{R}^{2 n+d}$. Moreover, all differential objects will be assumed to be of class $C^{\infty}$ if not otherwise stated.

The Newlander-Nirenberg Theorem [22] states that for $d=0$ the bundle $H^{1,0} M$ gives rise to a complex structure on $M$. We shall make repeated use of this fundamental result.

A CR manifold is locally embeddable (or realizable) if there exists a smooth embedding $\iota: M \rightarrow C^{n+d}$ such that

$$
\iota_{*} H^{0,1} M \subseteq\left\langle\frac{\partial}{\partial \bar{z}_{1}}, \ldots, \frac{\partial}{\partial \bar{z}_{n+d}}\right\rangle_{C},
$$

where $\langle\cdot, \ldots, \cdot\rangle_{\boldsymbol{C}}$ stands for the complex linear span. Not all CR manifolds are embeddable as was first shown by Nirenberg [23] for 3-dimensional CR manifolds. See also [14] and [21] for more general examples of non-embeddable CR manifolds (e.g., of higher codimension). To ensure embeddability one must therefore impose further (geometric or analytic) conditions on $M$. For example, if the CR structure on $M$ is real analytic, then $M$ is embeddable, see [1]. According to a theorem of Kuranishi, extended by Akahori and Webster (see [28] and references therein), every strictly pseudoconvex hypersurface-type CR manifold of dimension $\geq 7$ is locally realizable.

2000 Mathematics Subject Classification. Primary 32V30; Secondary 32M05. 
It turns out that a geometric condition implying embeddability (which makes no reference to the Levi form of $M$ ) is given by the presence of a transversal group action. Historically, 1-parameter groups of CR actions were first introduced by Tanaka [25]. Baouendi, Rothschild and Treves in [9] (see also [10]) proved that a CR manifold is embeddable if its CR structure is invariant under a transverse Lie group action. This result holds true even more generally for the so-called hypo-analytic structures [26], see also [13] for related results.

CR manifolds which admit a transverse abelian CR action are called rigid. Note, however, that the class of rigid CR manifolds is very restricted. In general, CR manifolds do not even allow any non-trivial Lie group action which preserves the real bundle $H M$. The aim of this paper is to give sufficient conditions for embeddability in terms of more general group actions that are not necessarily required to preserve the CR structure. We also show that the presence of such group action is a necessary condition for embeddability.

In Section 2 we introduce the concept of transverse extendable $\boldsymbol{R}^{d}$-actions on a CR manifold. These contain the abelian transverse actions in the sense of [9] as a special case. This notion is inspired by Lempert's definition of the so-called inner actions for 3-dimensional strictly pseudoconvex CR manifolds in [19]. There a special class of $S^{1}$-actions is studied and various (global) embedding results for CR 3-manifolds are obtained. Similar techniques were used by Bland and Duchamp [6] to investigate deformations of CR structures. We show that real analytic CR manifolds always admit extendable actions. The main result of this section is that the existence of an extendable action implies local embeddability.

Section 3 is devoted to CR manifolds of hypersurface type. For these we introduce the notion of semi-extendable action, which is a one-sided version of a local $\boldsymbol{R}$-action. We show that if a hypersurface type CR manifold admits a semi-extendable action, then it is locally embeddable as the boundary of a complex manifold. Conversely, it is shown that nondegenerate hypersurfaces in $\boldsymbol{C}^{n+1}$ admit a (local) semi-extendable $\boldsymbol{R}$-action. This is proved via a family of stationary disks locally attached to the hypersurface. For strictly pseudoconvex CR manifolds we obtain a somewhat sharper result.

Part of this paper was written while the second author was staying at Purdue University. He would like to thank the Mathematics Department for its hospitality and Professor László Lempert for numerous discussions on the subject treated in the paper. Part of this research was supported by Project 20-6137900 of Swiss National Science Foundation.

2. Analytic actions on CR manifolds. Let $M$ be a CR manifold of real dimension $2 n+d$ and $\operatorname{dim}_{C} H^{0,1} M=n$. Assume we are given a local $\boldsymbol{R}^{d}$-action

$$
F: M^{\prime} \times V \rightarrow M
$$

where $M^{\prime}$ is a neighborhood of $p \in M$ and $V=V_{p}$ is an open neighborhood of $0 \in \boldsymbol{R}^{d}$. To $\hat{X} \in T_{0} \boldsymbol{R}^{d}$ we assign a vector field $X$ which acts on $f \in C^{\infty}(M, \boldsymbol{R})$ by

$$
X f(p)=\left.\frac{d}{d t}\right|_{t=0} f(F(p, \exp t \hat{X})) .
$$


Denote $\mathcal{E}=\left\{X: \hat{X} \in T_{0} \boldsymbol{R}^{d}\right\}$. We will assume that the action is transverse, i.e.

$$
H_{p}^{1,0} M \oplus H_{p}^{0,1} M \oplus C \mathcal{E}_{p}=T_{p}^{C} M
$$

for each $p \in M$. For $s \in V$ we denote by

$$
H^{0,1}(s)=F(\cdot, s)^{*}\left(H^{0,1} M\right) \subseteq T^{C} M
$$

the pullback of $H^{0,1} M$ by $F(\cdot, s), s \in V . H^{0,1}(s)$ can be viewed as a point $\left[H^{0,1}(s)\right]$ in the Grassmannian $\operatorname{Gr}_{p}=\operatorname{Gr}\left(T_{p}^{C} M, n\right)$ of $n$-planes in $T_{p}^{C} M$. Let $\mathrm{Gr}$ be the bundle

$$
\mathrm{Gr}=\bigcup_{p \in M}\{p\} \times \mathrm{Gr}_{p} .
$$

Consider the mapping

$$
\begin{aligned}
\mu: \quad & M \times V \rightarrow \mathrm{Gr} \\
& (p, s) \mapsto\left[H_{p}^{0,1}(s)\right] .
\end{aligned}
$$

In the following definition we use the fact that $\mathrm{Gr}_{p}$ is a complex manifold (of dimension $n(n+d))$.

Definition 1. We say that the action $\left(F_{S}\right)$ is extendable at $p \in M$ if there exists a neighborhood $U \subseteq M$ of $p \in M$, a neighborhood $\tilde{V}$ of $0 \in C^{d}$ and a smooth mapping $\tilde{\mu}: U \times \tilde{V} \rightarrow \operatorname{Gr}$ such that $\left.\tilde{\mu}\right|_{U \times(\tilde{V} \cap V)}=\mu$ and $\tilde{\mu}(q, \cdot): \tilde{V} \rightarrow \mathrm{Gr}_{q}$ is holomorphic for each $q \in U$.

An immediate class of examples of CR manifolds that admit extendable $\boldsymbol{R}^{d}$-actions is that of the so-called rigid CR manifolds in the sense of [9], [10] which admit a CR action, i.e., $\mu(p, \cdot)$ is constant.

To specify the meaning of Definition 1 in local coordinates, observe that for small values of $s \in V$ the bundle $H^{0,1}(s)$ is close to $H^{0,1}(0)=H^{0,1} M$. There exists a $d$-parameter family of complex bundle morphisms

$$
\mu(s): H^{0,1} M \rightarrow H^{1,0} M \oplus C \mathcal{E}
$$

such that

$$
H^{0,1}(s)=\left\{\bar{Z}+\mu(\bar{Z}): \bar{Z} \in H^{0,1} M\right\} .
$$

(These expressions are defined only for small $s$.) If we choose a basis $Z_{1}, \ldots, Z_{n}$ of $H^{1,0} M$ and denote by $\theta_{1}, \ldots, \theta_{n}$ its dual basis, then $\mu$ is given by a tensor

$$
\mu=\mu_{i k} \bar{\theta}_{i} \otimes W_{k},
$$

where $W_{1}, \ldots, W_{n+d}=Z_{1}, \ldots, Z_{n}, X_{1}, \ldots, X_{d}$ and $X_{i}$ are the vector fields induced by the generators $\hat{X}_{i}=\partial / \partial s_{i}$. We will refer to $\mu$ as the Beltrami tensor. Let us mention that the tensor $\mu$ can be viewed as a generalization of the deformation tensor introduced in [6]. A similar definition, called complex dilatation, for maps between CR manifolds is given in [15] (see also [2]). Now we see that the $\boldsymbol{R}^{d}$-action $\left(F_{S}\right)$ is extendable in the sense of Definition 1 if all coefficients $\mu_{i k}$ of the Beltrami tensor are real analytic in $s \in \boldsymbol{R}^{d}$ and their radii of convergence have a uniform lower bound in a neighborhood of $p$. 
Proposition 1. Analytic CR manifolds admit local extendable actions.

Proof. Let $(u, s)=\left(u_{1}, \ldots, u_{2 n}, s_{1}, \ldots, s_{d}\right) \in \boldsymbol{R}^{2 n+d}$ be analytic coordinates of $M$ in a neighborhood of some point of $M$. In these coordinates we can write a basis of $H^{0,1} M$ as

$$
\bar{Z}_{j}=\alpha_{j k} \frac{\partial}{\partial u_{k}}+\beta_{j l} \frac{\partial}{\partial s_{l}}, \quad j=1, \ldots, n,
$$

where summation convention is used over repeated indices and the coefficients $\alpha_{j k}, \beta_{j l}$ are real analytic complex-valued functions of $(u, s) \in \boldsymbol{R}^{2 n+d}$. As $H M$ is real $2 n$-dimensional, we may assume that $\partial / \partial s_{1}, \ldots, \partial / \partial s_{d}$ are transverse to $H M$. The matrix $\left(\alpha_{j k}\right)$ has rank $n$ and so we may (by changing the basis) further assume that equations (6) read

$$
\bar{Z}_{j}=\frac{\partial}{\partial u_{j}}+a_{j k} \frac{\partial}{\partial v_{k}}+b_{j l} \frac{\partial}{\partial s_{l}}, \quad j=1, \ldots, n,
$$

where we use the notation $v_{k}=u_{n+k}, k=1, \ldots n$.

The local $\boldsymbol{R}^{d}$-action $\left(F_{S}\right)$ will be generated by the vector fields $\partial / \partial s_{1}, \ldots, \partial / \partial s_{d}$. Let $\Sigma$ be the submanifold $\Sigma=\{s=0\}$, parametrized by the coordinates $u \in \boldsymbol{R}^{2 n}$. We shall prove the extendability of the action $\left(F_{S}\right)$ at a point $p=(u, 0)$ on $\Sigma$. Our first observation is that the matrix $\operatorname{Im} a(u, 0)=\operatorname{Im}\left(a_{j k}(u, 0)\right)$ is invertible. To see this we argue as follows. Since $\left\{Z_{j}, \bar{Z}_{j}\right\}_{j=1, \ldots, n}$ forms a system of $2 n \boldsymbol{C}$-independent vectors, the system $\left\{V_{j}=(1 / 2 i)\left(Z_{j}-\right.\right.$ $\left.\left.\bar{Z}_{j}\right) ; j=1, \ldots, n\right\}$ is $\boldsymbol{R}$-independent in $H M$. Suppose that the real $n \times n$ matrix $\operatorname{Im} a\left(u_{0}, 0\right)$ is not invertible. Then a suitable linear combination with real coefficients $\left\{d_{j} ; j=1, \ldots, n\right\}$, of the rows from $\operatorname{Im} a\left(u_{0}, 0\right)$ will vanish. This means that the vector $V:=d_{j} V_{j}$ can be expressed as $V=e_{i}\left(\partial / \partial s_{i}\right)$ for some real coefficients $e_{i}, i=1, \ldots, d$. On the other hand, vectors $V_{j}, j=1, \ldots, n$, are $\boldsymbol{R}$-independent, which implies that $V \neq 0, V \in H_{\left(u_{0}, 0\right)} M$. This is in contradiction with the transversality of $\left\{\partial / \partial s_{1}, \ldots, \partial / \partial s_{d}\right\}$ and $H M$ at the point $\left(u_{0}, 0\right)$, which shows that $\operatorname{Im}\left(a_{j k}(u, 0)\right)$ is invertible.

Directly by the definition of the action $\left(F_{S}\right)$ we have

$$
F_{s}^{*} \bar{Z}_{j}\left(F_{s}(p)\right)=\frac{\partial}{\partial u_{j}}+a_{j k}(u, s) \frac{\partial}{\partial v_{k}}+b_{j l}(u, s) \frac{\partial}{\partial s_{l}} .
$$

Since we consider $u$ as fixed, we will for the rest of this proof suppress the dependence of $u$ and just write $a_{j k}(s)=a_{j k}(u, s)$ etc.

According to (5) the Beltrami tensor $\mu$ at $p$ with respect to the basis

$$
\bar{Z}_{1}(0), \ldots, \bar{Z}_{n}(0), \frac{\partial}{\partial s_{1}}, \ldots, \frac{\partial}{\partial s_{d}},
$$

can be written in the form

$$
\mu=\lambda_{i j} \bar{\theta}_{i} \otimes Z_{j}(0)+v_{i l} \bar{\theta}_{i} \otimes \frac{\partial}{\partial s_{l}} .
$$

We have to show that the coefficients $\lambda_{i j}, v_{i l}$ depend analytically on $s$. Let $X$ be the bundle spanned by $\partial / \partial s_{1}, \ldots, \partial / \partial s_{d}, \pi_{X}: T^{C} M \rightarrow H^{C} M$ be the projection along $X$, and $\pi^{0,1}$ : 
$H^{C} M \rightarrow H^{0,1} M$ be the canonical projection to $H^{0,1} M$. We have

$$
\pi^{0,1} \circ \pi_{X}\left(F_{s}^{*} \bar{Z}_{i}(s)\right)=c_{i j}(s) \bar{Z}_{j}(0)=\bar{W}_{i}(s) .
$$

By definition of the Beltrami tensor we have

$$
\bar{W}_{i}(s)+\mu\left(\bar{W}_{i}(s)\right)=F_{s}^{*} \bar{Z}_{i}(s) .
$$

Using equations (8) and (9), this reads

$$
\begin{aligned}
\frac{\partial}{\partial u_{i}} & +a_{i k}(s) \frac{\partial}{\partial v_{k}}+b_{i l}(s) \frac{\partial}{\partial s_{l}}=c_{i j}(s)\left(\frac{\partial}{\partial u_{j}}+a_{j k}(0) \frac{\partial}{\partial v_{k}}+b_{j l}(0) \frac{\partial}{\partial s_{l}}\right) \\
& +\lambda_{j k}(s) c_{i j}(s)\left(\frac{\partial}{\partial u_{k}}+\bar{a}_{k l}(0) \frac{\partial}{\partial v_{l}}+\bar{b}_{k m}(0) \frac{\partial}{\partial s_{m}}\right)+v_{j k}(s) c_{i j}(s) \frac{\partial}{\partial s_{k}} .
\end{aligned}
$$

Writing $a=\left(a_{i k}\right)$ etc. and comparing coefficients, we get the following system of matrix equations:

$$
\begin{aligned}
I & =c(s)+\lambda(s) c(s), \\
a(s) & =c(s) a(0)+\lambda(s) c(s) \bar{a}(0), \\
b(s) & =c(s) b(0)+\lambda(s) c(s) \bar{b}(0)+v(s) c(s) .
\end{aligned}
$$

From the first and second equations we get $c(s)(a(0)-\bar{a}(0))=a(s)-\bar{a}(0)$. Now we use the fact that $\operatorname{Im} a(0)$ is invertible; hence we conclude that $c(s)$ is analytic in $s$. From $c(0)=I$ we see that $c(s)$ is invertible (at least for small values of $s$ ) and hence $\lambda(s)=c^{-1}(s)-I$ is analytic in $s$. From the third equation we conclude that also $v(s)$ is analytic.

One can easily see that the above consideration is independent of the initial point. So we obtain that for each fixed $\left(u_{0}, s_{0}\right)$ the functions $\lambda\left(u_{0}, s_{0}+s\right), v\left(u_{0}, s_{0}+s\right)$ are real analytic in $s$. Moreover, the proof shows that the radii of convergence of the corresponding power series expansions have a locally in $\left(u_{0}, s_{0}\right)$-uniform lower bound which gives that $\left(F_{S}\right)$ is an extendable action.

Remark. Consider the following example: $M=\{\rho=0\} \subseteq \boldsymbol{C}^{2}$,

$$
\rho\left(x_{1}+i y_{1}, x_{2}+i y_{2}\right)=x_{1}^{4}+y_{1}^{4}+x_{2}^{2}+\left(y_{2}-1\right)^{2}-1 .
$$

$M$ is an analytic hypersurface in $\boldsymbol{C}^{2}$. Therefore it is an analytic CR manifold which admits an extendable $\boldsymbol{R}$-action according to Proposition 1. We claim that $M$ is not rigid in the sense of [9], since it does not admit a CR action. Indeed, $M$ does not even admit a transverse action preserving the real bundle $H M$ in a neighborhood of 0 . This follows from the fact that $H M$ is a contact bundle away from 0 , but the contact property fails at 0 . We refer to the next section for the formal definition of the contact property. An equivalent way to check the non-existence of a transverse action preserving $H M$ is as follows. Observe that vectorfields in $H M$ together with their first order commutators span the whole tangent space at a point $p \in M, p \neq 0$. However, this property fails exactly at the point $0 \in M$. This clearly contradicts the existence of a transverse $\boldsymbol{R}$-action preserving the bundle $H M$. 
We already mentioned in the Introduction that both rigid and analytic CR manifolds are embeddable ([9], [1]). A generalization of these results is our next statement.

THEOREM 1. If a CR manifold $M$ has an extendable local $\boldsymbol{R}^{d}$-action, then it is locally embeddable in $\boldsymbol{C}^{n+d}$.

Proof. Without loss of generality we can assume (see [26] p. 302, or Lemma I.1 in [9]) that points of $M$ are given in local coordinates $(u, s)=\left(u_{1}, \ldots, u_{2 n}, s_{1}, \ldots, s_{d}\right) \in \boldsymbol{R}^{2 n+d}$ such that

$$
X_{j}=\frac{\partial}{\partial s_{j}}, \quad j=1, \ldots, d
$$

induce the $\boldsymbol{R}^{d}$-action $\left(F_{S}\right)$ which is just the usual translation with respect to coordinates $s \in$ $\boldsymbol{R}^{d}$. Let $\Sigma \hookrightarrow M$ be the submanifold $\Sigma=\{s=0\}$ which is transverse to the action of $\left(F_{S}\right)$.

Choosing smooth sections $\bar{Z}_{1}(u), \ldots, \bar{Z}_{n}(u)$ of $\left.H^{0,1} M\right|_{\Sigma}$, we obtain by (5) at each point $(u, 0) \in \Sigma$ a Beltrami tensor $\mu(u, s)$ expressed with respect to the basis $\bar{Z}_{1}(u), \ldots, \bar{Z}_{n}(u)$, $X_{1}, \ldots, X_{d}$. As the action $\left(F_{s}\right)$ is extendable, each coefficient $\mu_{i j}(u, s)$ of the Beltrami tensor has an extension $\mu_{i j}(u, z), z_{i}=s_{i}+i t_{i}, i=1, \ldots, d$, which is holomorphic for $z$ in some neighborhood of 0 in $\boldsymbol{C}^{d}$. (To simplify the notation we will always write $\boldsymbol{C}^{d}$ where we actually mean an open neighborhood of 0 in $\boldsymbol{C}^{d}$.)

Next we define vector fields $\bar{W}_{i}$ on the manifold $N=\Sigma \times C^{d}$ by

$$
\bar{W}_{i}(u, z)=\bar{Z}_{i}(u)+\mu(u, z)\left(\bar{Z}_{i}(u)\right) .
$$

We understand the vectors $\bar{Z}_{i}(u)$ and $\mu(u, z)\left(\bar{Z}_{i}(u)\right)$ from the right side of (10) as being transported to the point $(u, z) \in N$.

Let us observe that for $\operatorname{Im} z=t=0$, by the definition of the Beltrami tensor, it follows that the vectors $W_{i}(u, s)$ form a basis of $H_{(u, s)}^{0,1} M$. Since $M$ is a CR manifold, the bundle $H^{0,1} M$ satisfies the integrability condition $\left[H^{0,1} M, H^{0,1} M\right] \subseteq H^{0,1} M$. It follows that there are smooth functions $c_{k}^{m, l}, k, l, m=1, \ldots, n$, such that

$$
\left[\bar{W}_{m}(u, s), \bar{W}_{l}(u, s)\right]=c_{k}^{m, l}(u, s) \bar{W}_{k}(u, s) .
$$

Next we show that the functions $c_{k}^{m, l}$ have holomorphic extensions to $\operatorname{Im} z \neq 0$ such that (11) holds also for $\operatorname{Im} z \neq 0$. Let

$$
\left(x_{1}, \ldots, x_{2 n+2 d}\right)=\left(u_{1}, \ldots, u_{2 n}, s_{1}, \ldots, s_{d}, t_{1}, \ldots, t_{d}\right) \in \boldsymbol{R}^{2 n+2 d}
$$

be real coordinates on $N$. Let us rewrite the vectors from (10) as

$$
\bar{W}_{i}(u, z)=a_{i j}(u, z) \frac{\partial}{\partial x_{j}}, \quad i=1, \ldots, n,
$$

where the coefficients $a_{i j}$ are holomorphic in $z \in C^{d}$ and $a_{i j} \equiv 0$ for the indices $j=$ $2 n+d+1, \ldots, 2 n+2 d$.

Let

$$
\bar{U}(u, z)=\left[\bar{W}_{m}(u, z), \bar{W}_{l}(u, z)\right]
$$


and write

$$
\bar{U}(u, z)=b_{j}(u, z) \frac{\partial}{\partial x_{j}} .
$$

Since the derivatives $\partial a_{i j}(u, z) / \partial x_{k}$ are holomorphic in $z$, it follows that also the coefficients $b_{j}$ are holomorphic functions in $z$ and $b_{j} \equiv 0, j=2 n+d+1, \ldots, 2 n+2 d$. By the previous consideration we know that for $z=s \in \boldsymbol{R}^{d}$ we have

$$
\bar{U}(u, s)=c_{j}(u, s) \bar{W}_{j}(u, s) .
$$

Writing $A=\left(a_{i j}\right), b=\left(b_{1}, \ldots, b_{2 n+d}\right)$ and $c=\left(c_{1}, \ldots, c_{n}\right)$, we obtain the matrix equation

$$
b=c A \text {. }
$$

As the vectors $\bar{W}_{i}$ are $C$-independent, there exists an invertible $n \times n$ submatrix $A_{1}$ of $A$, and submatrix $b_{1}$ of $b$ such that $b_{1}=c A_{1}$, from which we obtain $c=b_{1} A_{1}^{-1}$ as the required holomorphic extension $c(u, z)$. We also obtain immediately the extension of (11). This is seen as follows: The coefficients of the vector field

$$
\bar{U}(u, z)-c_{j}(u, z) \bar{W}_{j}(u, z)
$$

are holomorphic in $z$ and vanish on $\operatorname{Im} z=0$. But then they must vanish for all $z$. Consequently, (11) holds on the whole $N$.

We define on $N=\Sigma \times C^{d}$ the vector fields $\bar{V}_{i}=\partial / \partial \bar{z}_{i}, i=1, \ldots, d$. Clearly, $\left[\bar{V}_{i}, \bar{V}_{k}\right]=0$ and

$$
\left[\bar{V}_{i}, \bar{W}_{k}\right]=\frac{\partial}{\partial \bar{z}_{i}} \mu(u, z)\left(\bar{Z}_{k}(u)\right)=0,
$$

since the coefficients of $\mu$ are holomorphic in $z$. It follows that the bundle $\overline{\mathcal{N}}=$ $\left\langle\bar{W}_{1}, \ldots, \bar{W}_{n}, \bar{V}_{1}, \ldots, \bar{V}_{d}\right\rangle_{\boldsymbol{C}}$ satisfies the integrability condition

$$
[\overline{\mathcal{N}}, \overline{\mathcal{N}}] \subseteq \overline{\mathcal{N}}
$$

Moreover, it is easy to check that $\mathcal{N} \cap \overline{\mathcal{N}}=\{0\}$. It follows from the Newlander-Nirenberg Theorem that $\mathcal{N}$ gives rise to a complex structure on the manifold $N$ and that $M=\Sigma \times \boldsymbol{R}^{d}$ is CR embedded in $N$.

3. CR manifolds of hypersurface type. In this section we focus on hypersurfacetype CR manifolds $M=M^{2 n+1}$. Let us recall some notation that will be used throughout this section.

The Levi form of $M$ (see [7], p. 156) is given by

$$
\begin{aligned}
\mathcal{L}: & H^{1,0} M \rightarrow T M / H M \\
& \mathcal{L}(Z)=\pi((1 / 2 i)[Z, \bar{Z}]),
\end{aligned}
$$

where $\pi$ is the projection $\pi: T M \rightarrow T M / H M$. Choosing a Hermitian structure on $T^{C} M$ such that $H^{1,0} M$ is normal to $H^{0,1} M$, we can view $\mathcal{L}$ as a Hermitian quadratic form. $M$ is called non-degenerate if $\mathcal{L}$ is a non-degenerate form and strongly pseudoconvex if $\mathcal{L}(Z)$ is non-zero for $Z \neq 0$. If $M$ is non-degenerate, the bundle $H M=\operatorname{Re}\left(H^{1,0} M \oplus H^{0,1} M\right)$ is a 
contact distribution on $M$, i.e., there exists a 1 -form $\alpha$ on $M$ such that $H M=\operatorname{ker} \alpha$ and $\alpha$ is non-degenerate in the sense that

$$
\alpha \wedge d \alpha^{n} \neq 0 .
$$

For hypersurface-type CR manifolds we will consider semi-extendable actions rather than extendable actions. To be more precise, let $\left\{F_{s} ; s \in \boldsymbol{R}\right\}$ be a local $\boldsymbol{R}$-action that is transverse, i.e., the vector field $\left.\left(d F_{S} / d s\right)\right|_{s=0}$ is transverse to the contact field $H M$. (For the sake of simplicity we write $\boldsymbol{R}$ meaning a neighborhood of 0 in $\boldsymbol{R}$.) As in Definition 1 we can define the mapping

$$
\mu: M \times \boldsymbol{R} \rightarrow \mathrm{Gr}
$$

Let for $\varepsilon>0$

$$
B_{\varepsilon}=\{|z|<\varepsilon ; \operatorname{Im} z<0\} \subseteq C
$$

be the half disk in the lower half-plane of $\boldsymbol{C}$. We say that the action $g_{t}$ on $M$ is a semiextendable action in a neighborhood $U$ of some point $p \in M$ if there exists an $\varepsilon>0$ such that the mapping

$$
\mu: U \times(-\varepsilon, \varepsilon) \rightarrow \mathrm{Gr}
$$

extends to a smooth map on $U \times \bar{B}_{\varepsilon}$ that is holomorphic on $B_{\varepsilon}$ for each fixed $q \in U$. This definition is inspired by Lempert's definition of inner $S^{1}$-actions on 3-dimensional CR manifolds (see [19], [2]). Clearly, any extendable action in the sense of Definition 1 is a semi-extendable action. The proof of Theorem 1 can easily be modified to a proof of

THEOREM 2. If a hypersurface-type CR manifold $M$ admits a local semi-extendable $\boldsymbol{R}$-action, then it is locally realizable as the boundary of a complex manifold.

In the above statement $M$ is not necessarily required to be non-degenerate. In the case of hypersurface type $\mathrm{CR}$ manifolds that are pseudoconvex we have a more exact statement. We first have to introduce the notion of positivity of an action. Let $M$ be strictly pseudoconvex and $\left(F_{S}\right)$ be a transverse local $\boldsymbol{R}$-action inducing the vector field $S=\left.\left(d F_{S} / d s\right)\right|_{s=0}$ on $M$. We call the action positive if for $Z \in H^{1,0} M$ we have

$$
\frac{1}{2 i}[Z, \bar{Z}]=c(\bar{Z}) S \bmod H M
$$

for $c(\bar{Z})>0$.

THEOREM 3. If $M$ is strictly pseudoconvex and admits a positive and semi-extendable local $\boldsymbol{R}$-action, then $M$ can be locally realized as a strictly pseudoconvex hypersurface in $\boldsymbol{C}^{n+1}$.

In view of the embedding results due to Kuranishi, Akahori and Webster [28], mentioned in the Introduction, the above Theorem is significant mainly for $n=1$ and $n=2$. In higher dimensions pseudoconvexity alone implies embeddability. We will, however, apply Theorem 3 to prove that (independently of the dimension) embeddability as a strictly pseudoconvex hypersurface is equivalent to the presence of a semi-extendable action (see Theorem 4 below). 
Proof of THEOREM 3. As in the proof of Theorem 1, we can consider local coordinates in $M$ and the extension $N=\Sigma \times \boldsymbol{C}$ with coordinates $(u, z)$. Writing $z=s+i t \in \boldsymbol{C}$, the submanifold $\Sigma$ of $M$ is given by $\Sigma=\{s=0\}$. The vector field of the action is $S=\left.(\partial / \partial s)\right|_{M}$ and the vector fields $\bar{V}$ and $\bar{W}_{i}$ spanning $T^{0,1} N$ are given by $\bar{V}=\partial / \partial \bar{z}$ and

$$
\bar{W}_{i}(u, z)=\bar{Z}_{i}(u)+\mu(u, z)\left(\bar{Z}_{i}(u)\right), \quad i=1, \ldots, n,
$$

where the Beltrami tensor $\mu$ is holomorphic in $z \in \boldsymbol{H}^{-}=\{z \in \boldsymbol{C}$; $\operatorname{Im} z<0\}$. (Again, all expressions in this proof are to be read locally.)

Denote by $\overline{\mathcal{W}}$ the bundle spanned by $\bar{W}_{1}, \ldots, \bar{W}_{n}$. Using the same reasoning as in the proof of Theorem 1, one can use the semi-extendability of the given $\boldsymbol{R}$-action to show that $\overline{\mathcal{W}} \oplus \boldsymbol{C} \bar{V}$ defines a complex structure on $N^{-}=\Sigma \times \boldsymbol{H}^{-}$.

We next show that $M$ is pseudoconvex as seen from $N^{-}$. Pick a point $p \in \Sigma \hookrightarrow M$. We are going to construct a smooth function $\rho$ defined in a neighborhood of $p$ in $N$ that is strictly plurisubharmonic on $N^{-}$. We first need some preparations.

Recall that the Beltrami tensor at $p$ with respect to the basis $\bar{Z}_{1}, \ldots, \bar{Z}_{n}$ can be written in the form

$$
\mu=\lambda_{i j} \bar{\theta}_{i} \otimes Z_{j}+v_{i} \bar{\theta}_{i} \otimes S
$$

Since $\mu(u, 0)=0$ we have $\left.\bar{W}_{i}\right|_{\Sigma}=\bar{Z}_{i}$. The coefficients $v_{i}$ are holomorphic functions in $z \in \boldsymbol{H}^{-}$, and we denote

$$
\kappa=\max _{1 \leq i \leq n}\left|\frac{\partial}{\partial z} v_{i}(0)\right|
$$

Next, let $\|\cdot\|$ be the Hermitian metric on $\overline{\mathcal{W}}(p)$ such that $\bar{W}_{1}(p), \ldots, \bar{W}_{n}(p)$ form an orthonormal basis. By the positivity of the group action we know that for $\bar{W} \in \mathcal{W}$ we have

$$
\frac{1}{2 i}[W, \bar{W}]_{p}=c(\bar{W}) S(p) \quad \bmod \mathcal{W} \oplus \overline{\mathcal{W}}
$$

for some number $c(\bar{W})>0$. Let $c_{1}>0$ be a real number such that

$$
c(\bar{W}) \geq c_{1}\|\bar{W}\|^{2} .
$$

Finally, we set $A=n \kappa c_{1}^{-1}$. We define $\rho$ by

$$
\rho(u, z)=\rho\left(u_{1}, \ldots, u_{n}, s+i t\right)=A t^{2}+t .
$$

Clearly, we have $M=\{\rho=0\}$ and $\left.d \rho\right|_{M} \neq 0$. We show next that $\rho$ is strictly plurisubharmonic at $p$ (and hence on some neighborhood in $N^{-}$). This means that for sections $Y_{1}, Y_{2}$ of $T^{0,1} N$ the Hermitian form $\partial \bar{\partial} \rho\left(Y_{1}, \bar{Y}_{2}\right)$ is positive definite at $p$. We will make repeated use of the formula

$$
\partial \bar{\partial} \rho\left(Y_{1}, \bar{Y}_{2}\right)=Y_{1}\left(\bar{Y}_{2} \rho\right)-\bar{\partial} \rho\left(\left[Y_{1}, \bar{Y}_{2}\right]\right),
$$

which is easily verified (cf. Proposition 5.2 in [19]).

We have to show that $\partial \bar{\partial} \rho(Y, \bar{Y})>0$ for a non-zero local section $Y \in T^{1,0} N$. Without loss of generality we may assume that $Y$ is of the form

$$
Y=W+b V
$$


where $W=\sum a_{i} W_{i} \in \mathcal{W}$ and the coefficients $a_{i}, b$ are constant. We have

$$
\begin{aligned}
\partial \bar{\partial} \rho(Y, \bar{Y})= & \partial \bar{\partial} \rho(W, \bar{W})+|b|^{2} \partial \bar{\partial} \rho(V, \bar{V}) \\
& +2 \operatorname{Re} \partial \bar{\partial} \rho(b V, W)=(\mathrm{i})+(\text { ii })+(\text { iii }) .
\end{aligned}
$$

The term (ii) is easily calculated. Putting $Y_{1}=Y_{2}=V$ in formula (16), we get

$$
\partial \bar{\partial} \rho(V, \bar{V})=V \bar{V} \rho=-\frac{1}{2} \frac{\partial^{2}}{\partial z \partial \bar{z}}\left(A(z-\bar{z})^{2}+i(z-\bar{z})\right)=A \text {. }
$$

To calculate the term (i) in (17) write $W=W_{1}-i J W_{1}$, where $W_{1}, J W_{1}$ are real vector fields and $J$ is the complex tensor in $\mathcal{W} \oplus \overline{\mathcal{W}}$. Since $[W, \bar{W}]=2 i\left[W_{1}, J W_{1}\right]$, the positivity of the $\boldsymbol{R}$-action implies that

$$
\left[W_{1}, J W_{1}\right]=c(\bar{W}) S \bmod \mathcal{W} \oplus \overline{\mathcal{W}} .
$$

We have (at the point $p$ )

$$
\bar{\partial} \rho(S)=\frac{1}{2} d \rho(S+i J S)=\frac{i}{2}(J S) \rho=\frac{i}{2} \frac{\partial}{\partial t} \rho=\frac{i}{2} .
$$

Note that $W$ and $\bar{W}$ are tangent to the level sets $\rho=$ constant. Using formula (16), we arrive at

$$
\begin{aligned}
\partial \bar{\partial} \rho(W, \bar{W}) & =-\bar{\partial} \rho([W, \bar{W}]) \\
& =-\bar{\partial} \rho\left(2 i\left[W_{1}, J W_{1}\right]\right)=-2 i c(\bar{W}) \bar{\partial} \rho(S)=c(\bar{W}) .
\end{aligned}
$$

By (14) we get the estimate

$$
\partial \bar{\partial} \rho(W, \bar{W}) \geq c_{1} \sum\left|a_{i}\right|^{2} .
$$

It remains to estimate the term (iii) in (17). Observe that

$$
[V, \bar{W}]_{p}=\left(\frac{\partial}{\partial z} \mu\right)(\bar{W}) \equiv \beta S \quad \bmod \mathcal{W} \oplus \overline{\mathcal{W}}
$$

where $\beta=\sum \bar{a}_{i}\left(\partial v_{i} / \partial z\right)(0)$. Putting $Y_{1}=V$ and $Y_{2}=W$ in (16), we get

$$
\partial \bar{\partial} \rho(V, \bar{W})=-\bar{\partial} \rho([V, \bar{W}])=-\beta \bar{\partial} \rho(S)=-\frac{i}{2} \beta .
$$

From this and the definition of $\kappa$ (see (13)) we obtain the estimate

$$
|\partial \bar{\partial} \rho(b V, \bar{W})| \leq \frac{\kappa}{2}|b| \sum\left|a_{i}\right| .
$$

Inserting (18), (19) and (20) in (17), we obtain

$$
\begin{aligned}
\partial \bar{\partial} \rho(Y, \bar{Y}) & \geq c_{1} \sum\left|a_{i}\right|^{2}+A|b|^{2}-\kappa|b| \sum\left|a_{i}\right| \\
& =c_{1} \sum\left|a_{i}\right|^{2}+n\left(\kappa^{2} / c_{1}\right)|b|^{2}-\kappa|b| \sum\left|a_{i}\right| \\
& =c_{1}\left(\sum\left(\left|a_{i}\right|^{2}+\left(\kappa^{2} / c_{1}^{2}\right)|b|^{2}-\left(\kappa / c_{1}\right)\left|a_{i} \| b\right|\right)\right) .
\end{aligned}
$$

It is easy to see that the last expression is strictly positive for $Y \neq 0$.

We have shown that $M$ is a strictly pseudoconvex piece of the boundary of the complex manifold $N^{-}$. By a theorem of Catlin [11] it is possible to locally extend the complex structure 
to $N=\Sigma \times C$. In this way we obtain a local embedding of $M$ in $C^{n+1}$. This proves Theorem 3.

The rest of this section is devoted to proving a partial converse to Theorem 3 . We have

THEOREM 4. Let $M=M^{2 n+1}$ be an embedded hypersurface in $C^{n+1}$, which is nondegenerate at the point $p \in M$. Then $M$ admits a semi-extendable $\boldsymbol{R}$-action in a neighborhood of $p$.

A major component in the proof of this theorem is the following proposition. To formulate the statement let us recall that a (real) $N$-dimensional submanifold $S$ of complex $N$-space $\mathcal{C}$ is called totally real at a point $p \in S$ if

$$
T_{p} C^{N}=T_{p} S \oplus J T_{p} S
$$

where $J$ is the complex structure of $\mathcal{C}$.

Denote by $\Delta$ the unit disk in $C$ and by $\gamma$ the subarc $\left\{e^{i s} ; s \in(-\pi / 2, \pi / 2)\right\}$ of $\partial \Delta$.

Proposition 2. Let $S \hookrightarrow C^{N}$ be a smooth manifold, totally real at $p \in S$, and let $X \in T_{p} S$. Then there exists a smooth family $F_{r}, r \in U \subseteq R^{N-1}$, of maps $F_{r}: \bar{\Delta} \rightarrow C^{N}$, analytic in $\Delta$, such that

1. $F_{0}(1)=p$ and $\left.(d / d s)\right|_{s=0} F_{0}\left(e^{i s}\right)=\lambda X$ for some $\lambda>0$;

2. the family of curves $\left\{F_{r}(\gamma), r \in U\right\}$ smoothly foliates a neighborhood of $p$ in $S$.

PROOF. The proof is done in two steps. In the first step we establish the existence of the disc family required in the statement in a smoothness class $C^{k, \alpha}$ for a fixed $k \in N$ and $0<\alpha<1$. The second step is a bootstrapping arguement yielding $C^{\infty}$ regularity of foliation.

SteP 1: EXISTENCE. This step is based on a Bishop-type argument. For an introduction to the Bishop equation and related machinery we refer to [7]. Our proof is modeled after that of Pinchuk in [24].

Let us assume that $p=0$. Since $S$ is totally real at $0 \in S$, it can locally be represented after a biholomorphic change of variables in the form

$$
S=\{z=x+i y ; y=h(x)\},
$$

where $h: \boldsymbol{R}^{N} \rightarrow \boldsymbol{R}^{N}$ is a smooth function with $h(0)=0$ and $d h(0)=0$.

Let $k \in N, 0<\alpha<1$. For a function $x \in C^{k, \alpha}\left(\partial \Delta, \boldsymbol{R}^{N}\right)$ we shall consider the Hilbert transform defined by

$$
T x(z)=\operatorname{Im}\left\{\frac{1}{2 \pi i} \int_{|\zeta|=1}\left(\frac{\zeta+z}{\zeta-z}\right) x(\zeta) \frac{d \zeta}{\zeta}\right\}
$$

Note that the Hilbert transform $T: C^{k, \alpha}\left(\partial \Delta, \boldsymbol{R}^{N}\right) \rightarrow C^{k, \alpha}\left(\partial \Delta, \boldsymbol{R}^{N}\right)$ is a bounded linear map and $(x+i T x)(\zeta)$ is the restriction to $\partial \Delta$ of a holomorphic map from $\Delta$ into $\boldsymbol{C}^{N}$.

We make use of the implicit function theorem for Banach space $C^{k, \alpha}\left(\partial \Delta, \boldsymbol{R}^{N}\right)$. Consider a fixed smooth function $u: \partial \Delta \rightarrow \boldsymbol{R}$ with the properties $\left.u\right|_{\gamma} \equiv 0$ and $\left.u\right|_{\partial \Delta-\bar{\gamma}}<0$. We set up 
the following "ansatz" for the implicit function theorem:

$$
\begin{aligned}
\Phi: & \boldsymbol{R}^{N} \times \boldsymbol{R}^{N} \times C^{k, \alpha}\left(\partial \Delta, \boldsymbol{R}^{N}\right) \rightarrow C^{k, \alpha}\left(\partial \Delta, \boldsymbol{R}^{N}\right), \\
& \Phi(c, t, x)=x+T(h \circ x+t u)-c .
\end{aligned}
$$

(Here $t u=\left(t_{1} u, \ldots, t_{N} u\right)$.) Since $h$ is $C^{\infty}$, it is clear that $\Phi$ is smooth.

Clearly, we have $\Phi(0,0,0)=0$. Using the fact that $d h(0)=0$, we find that

$$
\nabla_{x} \Phi(0,0,0) X=X+T(d h(0) X)=X .
$$

We hence find a family of functions $x(c, t)$ in $C^{k, \alpha}\left(\partial \Delta, \boldsymbol{R}^{N}\right)$ satisfying

$$
\Phi(c, t, x(c, t))=0 .
$$

We set $f(c, t)(\zeta)=x(c, t)(\zeta)+i(h \circ x(c, t)(\zeta)+t u(\zeta))$. Since

$$
T(\operatorname{Im} f(c, t))=-\operatorname{Re} f(c, t)+c,
$$

the function $f(c, t)$ can be extended to $f(c, t): \bar{\Delta} \rightarrow C^{N}$ of class $C^{k, \alpha}$, which is holomorphic in $\Delta$. Moreover, for $\zeta \in \gamma$ we have $u(\zeta)=0$,

$$
\operatorname{Im} f(c, t)(\zeta)=h(\operatorname{Re} f(c, t)(\zeta)),
$$

and hence $f(c, t)(\gamma) \subseteq S$.

The disc family $\left(F_{r}\right)_{r}$ satisfying Conditions 1 and 2 from the statement will be a subfamily of $(f(c, t))_{(c, t)}$. The remaining part of the first step is devoted to the selection of this subfamily.

Let us introduce the mapping $G: \boldsymbol{R}^{N} \times \boldsymbol{R}^{N} \rightarrow \boldsymbol{R}^{N} \times \boldsymbol{R}^{N}$ by

$$
G(c, t)=\left(\begin{array}{l}
G_{1}(c, t) \\
G_{2}(c, t)
\end{array}\right)=\left.\left(\begin{array}{l}
x(c, t)\left(e^{i s}\right) \\
(d / d s) x(c, t)\left(e^{i s}\right)
\end{array}\right)\right|_{s=0} .
$$

We shall prove that $G$ is a local diffeomorphism in a neighborhood of the point $(0,0) \in$ $\boldsymbol{R}^{N} \times \boldsymbol{R}^{N}$. To do that we have to show that $D G(0,0)$ is invertible.

Let us first calculate the matrices $D_{c} G_{1}(0,0)$ and $D_{c} G_{2}(0,0)$. From (23) it follows that

$$
0=x(c, t)\left(e^{i s}\right)+T(h(x(c, t))+t u)\left(e^{i s}\right)-c .
$$

Differentiating (25) with respect to $c$ and setting $(c, t)=(0,0)$, we obtain

$$
0=D_{c} x(0,0)\left(e^{i s}\right)+T\left(d h(x(0,0)) D_{c} x(0,0)\right)\left(e^{i s}\right)-I .
$$

Since $x(0,0)=0$ and $d h(0)=0$, it follows that $D_{c} x(0,0)\left(e^{i s}\right)=I$, which implies that

$$
D_{c} G_{1}(0,0)=I \quad \text { and } \quad D_{c} G_{2}(0,0)=0 .
$$

In the next step we prove that $D_{t} G_{2}(0,0)$ is invertible. We differentiate now (25) with respect to $t$ and set again $(c, t)=(0,0)$. We then obtain

$$
D_{t} x(0,0)\left(e^{i s}\right)=T(I u)\left(e^{i s}\right),
$$

which implies that $D_{t} G_{2}(0,0)=\left.(d / d s)\right|_{s=0} T(I u)\left(e^{i s}\right)$. 
Using the fact that $\left.u\right|_{\gamma} \equiv 0$, the derivative $\left.(d / d s)\right|_{s=0} T u\left(e^{i s}\right)$ can be explicitly calculated by formula (21). The result of the calculation gives

$$
\left.\frac{d}{d s}\right|_{s=0} T u\left(e^{i s}\right)=\frac{1}{2 \pi} \int_{\pi / 2}^{3 \pi / 2} \frac{u\left(e^{i \theta}\right)}{\cos \theta-1} d \theta .
$$

As $u \equiv 0$ on $\gamma$ and $u<0$ on $\partial \Delta-\bar{\gamma}$, we get that (27) is positive. Hence

$$
D_{t} G_{2}(0,0)=\left.\frac{d}{d s}\right|_{s=0} T(u I)\left(e^{i s}\right)
$$

is invertible, which together with (26) implies that $G$ is a local diffeomorphism at $(0,0) \in$ $\boldsymbol{R}^{N} \times \boldsymbol{R}^{N}$.

Let $X \in T_{0} S=\boldsymbol{R}^{N} \times\{0\} \simeq \boldsymbol{R}^{N}$. By choosing $\lambda>0$ small enough, we conclude that there exists $\left(c_{0}, t_{0}\right)$ in a neighborhood of $(0,0)$ such that $G\left(c_{0}, t_{0}\right)=(0, \lambda X)$.

Let $H$ be the hyperplane in $\boldsymbol{R}^{N}$ orthogonal to $X$ and denote by

$$
H^{\prime}=D_{c}^{-1} x\left(c_{0}, t_{0}\right)(1)(H) \text {. }
$$

Consider a linear mapping $A: \boldsymbol{R}^{N-1} \rightarrow \boldsymbol{R}^{N}$ with the property that $A\left(\boldsymbol{R}^{N-1}\right)=H^{\prime}$. We claim that for a sufficiently small neighborhood $U$ of $0 \in \boldsymbol{R}^{N-1}$ the disc family $\left(F_{r}\right)_{r \in U}$ given by

$$
F_{r}(z)=f\left(c_{0}+A r, t_{0}\right)(z), \quad r \in U, z \in \Delta,
$$

satisfies Conditions 1 and 2 from the statement (except $C^{\infty}$ regularity).

Indeed, we can see immediately that Condition 1 is satisfied. To check Condition 2 , consider for small $\varepsilon>0$ the mapping

$$
H: U \times(-\varepsilon, \varepsilon) \rightarrow \boldsymbol{R}^{N}, \quad H(r, s)=x\left(c_{0}+A r, t_{0}\right)\left(e^{i s}\right) .
$$

It follows that

$$
D H(0,0)=\left(\begin{array}{l}
D_{c} x\left(c_{0}, t_{0}\right)(1) A \\
\lambda X
\end{array}\right) .
$$

By the definition of $A$ we see that the matrix $D H(0,0)$ is invertible and Condition 2 follows now easily by another application of the inverse function theorem. This concludes the first step in the proof of Proposition 2.

In conclusion we obtain the existence of the disc family required in the statement in the regularity class $C^{k, \alpha}\left(\partial \Delta, \boldsymbol{R}^{N}\right)$ for arbitrary, but fixed $k \in N$. This is not enough to claim $C^{\infty}$ regularity, since it could happen that the paremeter space for our disc family depends on $k$ and it shrinks to a point if $k \rightarrow \infty$. We deal with this difficulty in the following

SteP 2: Regularity. In this step we increase the regularity of the foliation obtained in the first step by a bootstrapping argument. We start with some preparations. We shall use the Hölder spaces $C_{\varepsilon}^{k, \alpha}\left(\partial \Delta, \boldsymbol{R}^{N}\right)$ obtained by changing the norm in $C^{k, \alpha}\left(\partial \Delta, \boldsymbol{R}^{N}\right)$ to the norm that depends on the parameter $1>\varepsilon>0$ according to the formula

$$
\|x\|_{k, \alpha, \varepsilon}:=\max _{0 \leq j \leq k}\left\{\varepsilon^{j}\left(\left\|x^{(j)}\right\|_{0}+\left\|x^{(j)}\right\|_{\alpha}\right)\right\},
$$


where $x^{(j)}$ is the $j$-th derivative of $x$,

$$
\left\|x^{(j)}\right\|_{0}=\sup _{\zeta \in \partial \Delta}\left\|x^{j}(\zeta)\right\| \quad \text { and } \quad\left\|x^{(j)}\right\|_{\alpha}=\sup _{\zeta_{1} \neq \zeta_{2}} \frac{\left\|x^{(j)}\left(\zeta_{1}\right)-x^{(j)}\left(\zeta_{2}\right)\right\|}{\left|\zeta_{1}-\zeta_{2}\right|^{\alpha}} .
$$

Let us recall that according to Privalov's theorem (see, eg., [8]) the Hilbert transform

$$
T: C^{0, \alpha}\left(\partial \Delta, \boldsymbol{R}^{N}\right) \rightarrow C^{0, \alpha}\left(\partial \Delta, \boldsymbol{R}^{N}\right)
$$

is a bounded linear operator such that

$$
\|T u\|_{0}+\|T u\|_{\alpha} \leq C\left(\|u\|_{0}+\|u\|_{\alpha}\right) \text { for } u \in C^{0, \alpha}\left(\partial \Delta, \boldsymbol{R}^{N}\right) .
$$

Let $a: \partial \Delta \rightarrow M_{N}(\boldsymbol{R})$ be a matrix valued $C^{k, \alpha}$ function and consider the linear operator $v \rightarrow Z v, Z v=T(a v)$. Using Privalov's theorem it follows that

$$
Z: C^{k, \alpha}\left(\partial \Delta, \boldsymbol{R}^{N}\right) \rightarrow C^{k, \alpha}\left(\partial \Delta, \boldsymbol{R}^{N}\right)
$$

is a bounded operator; moreover, $\|Z\|_{k, \alpha}<1$ if $\|a\|_{k, \alpha}$ is small enough. If we work in the weighted spaces $C_{\varepsilon}^{k, \alpha}\left(\partial \Delta, \boldsymbol{R}^{N}\right)$, we have a better statement as formulated in the following

CLAIM. There exists a constant $\delta>0$ such that if

$$
2\|a\|_{0}+\|a\|_{\alpha}<\delta,
$$

then for $k \in N$ we can choose $\varepsilon=\varepsilon(k, a)>0$ such that

$$
Z: C_{\varepsilon}^{k, \alpha}\left(\partial \Delta, \boldsymbol{R}^{N}\right) \rightarrow C_{\varepsilon}^{k, \alpha}\left(\partial \Delta, \boldsymbol{R}^{N}\right)
$$

becomes a contraction.

Indeed, using Privalov's theorem, write

$$
\begin{aligned}
\|Z v\|_{k, \alpha, \varepsilon} & =\max _{0 \leq j \leq k} \varepsilon^{j}\left(\left\|(T a v)^{(j)}\right\|_{0}+\|(\text { Tav })^{(j)} \|_{\alpha}\right) \\
& =\max _{0 \leq j \leq k} \varepsilon^{j}\left(\left\|T(a v)^{(j)}\right\|_{0}+\left\|T(a v)^{(j)}\right\|_{\alpha}\right) \\
& \leq C \cdot \max _{0 \leq j \leq k} \varepsilon^{j}\left(\left\|(a v)^{(j)}\right\|_{0}+\left\|(a v)^{(j)}\right\|_{\alpha}\right) .
\end{aligned}
$$

Without loss of generality we can assume that $N=1$. Using the product formula for higher derivatives

$$
(a v)^{(j)}=\sum_{i=0}^{j}\left(\begin{array}{l}
j \\
i
\end{array}\right) a^{(i)} v^{(j-i)},
$$

we can estimate the first part on the far right hand side of (28) as

$$
\begin{aligned}
\left\|\varepsilon^{j}(a v)^{(j)}\right\|_{0} & \leq\|a\|_{0} \cdot \varepsilon^{j}\left\|v^{(j)}\right\|_{0}+\varepsilon \cdot\left(\sum_{i=1}^{j}\left(\begin{array}{l}
j \\
i
\end{array}\right)\left\|a^{(i)}\right\|_{0}\right) \cdot \varepsilon^{j-i}\left\|v^{(j-i)}\right\|_{0} \\
& \leq\left(\|a\|_{0}+\varepsilon \cdot C_{1}(k, a)\right) \cdot\|v\|_{k, \alpha, \varepsilon}
\end{aligned}
$$

for all $j \leq k$.

To estimate the second part on the far right hand side of (28), we use that for $u_{1}, u_{2} \in C^{\alpha}$ we have

$$
\left\|u_{1} \cdot u_{2}\right\|_{\alpha} \leq\left\|u_{1}\right\|_{0} \cdot\left\|u_{2}\right\|_{\alpha}+\left\|u_{2}\right\|_{0} \cdot\left\|u_{1}\right\|_{\alpha} .
$$


Then it follows that

$$
\begin{aligned}
\left\|\varepsilon^{j}(a v)^{(j)}\right\|_{\alpha} & =\varepsilon^{j}\left\|\sum_{i=0}^{j}\left(\begin{array}{l}
j \\
i
\end{array}\right) a^{(i)} v^{(j-i)}\right\|_{\alpha} \\
& \leq \varepsilon^{j} \sum_{i=0}^{j}\left(\begin{array}{c}
j \\
i
\end{array}\right)\left(\left\|a^{(i)}\right\|_{0}\left\|v^{(j-i)}\right\|_{\alpha}+\left\|a^{(i)}\right\|_{\alpha}\left\|v^{(j-i)}\right\|_{0}\right) \\
& \leq\left(\|a\|_{0}+\|a\|_{\alpha}+\varepsilon \cdot C_{2}(k, a)\right)\|v\|_{k, \alpha, \varepsilon} .
\end{aligned}
$$

By a combination of (28), (29) and (30) the choices of

$$
\delta:=\frac{1}{2 C} \quad \text { and } \quad \varepsilon:=\frac{1}{2 C\left(C_{1}(k, a)+C_{2}(k, a)\right)}
$$

finish the proof of the claim.

Let us now fix a value of $k \geq 2$ and consider the family

$$
F: U \rightarrow C^{k, \alpha}\left(\partial \Delta, \boldsymbol{R}^{N}\right), \quad U \subseteq \boldsymbol{R}^{N-1},
$$

of discs obtained in the first part of the proof. Recall that for $r \in U$ the disc $F_{r}=x_{r}$ comes from the implicit function theorem related to the equation (22). We can write this equation as $\Psi(r, x)=0$, where

$$
\Psi(r, x)=x+T\left(h \circ x+t_{0} u\right)-\left(c_{0}+A r\right)
$$

with $A: \boldsymbol{R}^{N-1} \rightarrow \boldsymbol{R}^{2 N}$ a fixed linear mapping and $u: \partial \Delta \rightarrow \boldsymbol{R}^{N}$ a given smooth function.

Let us fix a value $r \in U$ and consider the associated disc $x_{r} \in C^{k, \alpha}\left(\partial \Delta, \boldsymbol{R}^{N}\right)$. To prove that $x_{r}^{\prime} \in C^{k, \alpha}\left(\partial \Delta, \boldsymbol{R}^{N}\right)$ we argue as follows. We differentiate (31) and obtain

$$
x_{r}^{\prime}+T\left(d h \circ x_{r} \cdot x_{r}^{\prime}\right)=-t_{0} T u^{\prime} .
$$

In other words, $v=x_{r}^{\prime}$ satisfies the equation

$$
(I+Z) v=-t_{0} T u^{\prime},
$$

where $Z v:=T\left(d h \circ x_{r} \cdot v\right)$. The operator $I+Z$ is invertible in $C_{\varepsilon}^{k, \alpha}\left(\partial \Delta, \boldsymbol{R}^{N}\right)$ if $Z$ is a contraction. Applying the above claim this follows provided

$$
2\left\|d h \circ x_{r}\right\|_{0}+\left\|d h \circ x_{r}\right\|_{\alpha}<\delta .
$$

Because $d h(0)=0$ it follows that $\left\|d h \circ x_{r}\right\|_{0}$ is small as long as $\left\|x_{r}\right\|_{0}$ is small. Furthermore, observe that

$$
\left\|d h \circ x_{r}\right\|_{\alpha} \leq 2^{1-\alpha}\left\|d^{2} h \circ x_{r}\right\|_{0} \cdot\left\|x_{r}^{\prime}\right\|_{0} .
$$

By the continuity of $r \rightarrow x_{r} \in C^{2, \alpha}$ we see that both $\left\|x_{r}\right\|_{0}$ and $\left\|x_{r}^{\prime}\right\|_{0}$ can be made sufficiently small if $r \in U^{\prime}$, where $U^{\prime} \subset U$ is a fixed parameter neighborhood.

Since $t \cdot T u^{\prime} \in C^{\infty}$, the invertibility of $I+Z$ yields that

$$
x_{r}^{\prime}=-(I+Z)^{-1}\left(t_{0} T u^{\prime}\right) \in C_{\varepsilon}^{k, \alpha}\left(\partial \Delta, \boldsymbol{R}^{N}\right),
$$

and so $x_{r} \in C^{k+1, \alpha}$. Iterating this reasoning, we obtain that $x_{r} \in C^{\infty}$ for $r \in U^{\prime}$. This proves the smoothness of each individual disc $x_{r}$. 
Smoothness of the foliation (i.e., dependence on the parameter $r \in U^{\prime}$ ) follows by the regularity of the implicit function $r \rightarrow x_{r}$ that is inherited from the smoothness of the mapping $\Psi: U \times C^{k, \alpha}\left(\partial \Delta, \boldsymbol{R}^{N}\right) \rightarrow C^{k, \alpha}\left(\partial \Delta, \boldsymbol{R}^{N}\right)$ for arbitrary $k \in N$. This concludes the proof of the proposition.

Proof of TheOrem 4. Using Proposition 2, we will first construct a family of "stationary" disks attached to $M$ along the half-circle $\gamma$. To do that we shall realize $M$ as a totally real submanifold in a higher dimensional complex space (cf. [3], [4]) as follows.

For a point $z \in M \hookrightarrow C^{n+1}$ let $H_{z} M$ be the maximal complex subspace of $T_{z} M$. Then $H_{z} M$ is a subspace of $\boldsymbol{C}^{n+1}$ of complex dimension $n$ and can hence be viewed as a point $\left[H_{z} M\right]$ in the projective space $C P^{n}$. Consider the map

$$
\begin{aligned}
\psi: & M \rightarrow C^{n+1} \times C P^{n} \\
& z \mapsto\left(z,\left[H_{z} M\right]\right) .
\end{aligned}
$$

We set $\mathcal{C}=\boldsymbol{C}^{n+1} \times C P^{n}$ and $S=\psi(M) \subseteq \mathcal{C}$. Furthermore, denote by $\pi$ the projection

$$
\pi: \mathcal{C} \rightarrow C^{n+1}
$$

to the first factor of $\mathcal{C}$.

According to a result of Webster [27] it follows that $S$ is a totally real manifold in $\mathcal{C}$ if $M$ is a strictly pseudoconvex hypersurface in $\boldsymbol{C}^{n+1}$. The following proposition is a more general version of this statement.

Proposition 3. Let $M \hookrightarrow C^{n+1}$ be a smooth hypersurface. Then $M$ is non-degenerate at $p \in M$ if and only if $S$ is totally real at $\left(p,\left[H_{p} M\right]\right) \in S$.

PROOF. Let us first observe that the statement is biholomorphically invariant. Therefore, by making an appropriate biholomorphic change of variables, we can assume (cf. [7], p. 208) that

$$
M=\left\{z=(x+i y, w) \in \boldsymbol{C} \times \boldsymbol{C}^{n} ; y=h(x, w)\right\},
$$

where $h: \boldsymbol{R} \times \boldsymbol{C}^{n} \rightarrow \boldsymbol{R}$ is a smooth function with the following Taylor expansion at the origin:

$$
h(x, w)=\sum q_{k}\left|w_{k}\right|^{2}+O(3), \quad q_{k} \in \boldsymbol{R}, k=1, \ldots, n .
$$

$M$ is non-degenerate at 0 if and only if $q_{k} \neq 0$ for $k=1, \ldots, n$. We shall show that this condition is also equivalent with $S$ being totally real at the point $\left(0,\left[H_{0} M\right]\right)$.

To obtain a local parametrization of $S$ in a neighborhood of $\left(0,\left[H_{0} M\right]\right)$ consider the defining function $\rho: \boldsymbol{C}^{n+1} \rightarrow \boldsymbol{R}$ of $M$ given by $\rho(z)=y-h(x, w)$. Then $M=\{z \in$ $\left.C^{n+1} ; \rho(z)=0\right\}$ and for $z \in M$ the complex tangent space $H_{z} M$ is given by

$$
H_{z} M=\left\{X \in \boldsymbol{C}^{n+1} ; \partial \rho(z) \cdot X=0\right\} .
$$

In the above expression $\partial \rho(z)$ denotes the vector

$$
\partial \rho(z)=\left(\frac{\partial \rho}{\partial u}, \frac{\partial \rho}{\partial w_{1}}, \ldots, \frac{\partial \rho}{\partial w_{n}}\right) \in \boldsymbol{C}^{n+1},
$$


where $u=x+i y$, and $A \cdot B$ denotes the complex multiplication of two vectors $A=$ $\left(A_{1}, \ldots, A_{n+1}\right), B=\left(B_{1}, \ldots, B_{n+1}\right), A \cdot B=\sum A_{j} B_{j}$.

From (33) we see that in a neighborhood $U$ of $0 \in C^{n+1}$ we have

$$
\partial \rho(z)=\left(-i / 2, q_{1} \bar{w}_{1}, \ldots, q_{n} \bar{w}_{n}\right)+O(2) .
$$

This implies that for $z \in U$ the point $\left[H_{z} M\right] \in \boldsymbol{C} P^{n}$ is represented by the vector

$$
[\partial \rho(z)]=\left(-2 i q_{1} \bar{w}_{1}, \ldots,-2 i q_{n} \bar{w}_{n}\right)+O(2) \in \boldsymbol{C}^{n} .
$$

From this we obtain the following local parametrization of $S$,

$$
S=\left\{\phi(x, w) ;(x, w) \in V \subseteq \boldsymbol{R} \times \boldsymbol{C}^{n}\right\},
$$

where $V$ is a neighborhood of $0 \in \boldsymbol{R} \times \boldsymbol{C}^{n}$, and $\phi: V \rightarrow \boldsymbol{C}^{2 n+1}$ is a smooth function which has the following expansion:

$$
\phi(x, w)=\left(x+i h(x, w), w_{1}, \ldots, w_{n},-2 i q_{1} \bar{w}_{1}, \ldots,-2 i q_{n} \bar{w}_{n}\right)+O(2) .
$$

Using this parametrization, we obtain that the tangent space of $S$ at $\left(0,\left[H_{0} M\right]\right)$ is given by

$$
T_{\left(0,\left[H_{0} M\right]\right)} S=\operatorname{span}_{\boldsymbol{R}}\left\{V_{j} \in C^{2 n+1} ; j=1, \ldots, 2 n+1\right\},
$$

where the vectors $V_{j}$ are the (real) partial derivatives of $\phi$.

Let us explicitly compute the vectors $V_{j}$ as follows:

$$
V_{1}=\frac{\partial \phi}{\partial x}(0,0)=(1,0, \ldots, 0) \in \boldsymbol{C}^{2 n+1} .
$$

Denoting by $w_{k}=x_{k}+i y_{k}, k=1, \ldots n$, we have

$$
\begin{gathered}
V_{1+k}=\frac{\partial \phi}{\partial x_{k}}(0,0)=\left(0, \ldots, 0,1,0, \ldots, 0,-2 i q_{k}, 0, \ldots, 0\right) \in \boldsymbol{C}^{2 n+1}, \\
V_{1+n+k}=\frac{\partial \phi}{\partial y_{k}}(0,0)=\left(0, \ldots, 0, i, 0, \ldots, 0,-2 q_{k}, 0, \ldots, 0\right) \in \boldsymbol{C}^{2 n+1} .
\end{gathered}
$$

Using these expressions, it is straightforward to check that the complex span of $\left\{V_{j} ; j=\right.$ $1, \ldots, 2 n+1\}$ is the whole $\boldsymbol{C}^{2 n+1}$ if and only if $q_{k} \neq 0$ for all $k=1, \ldots, n$. This concludes the proof of the proposition.

Proposition 3 makes it possible to apply Proposition 2 to prove the Theorem.

Let $p \in M$ and $P=\psi(p) \in S$. Choose a vector $\xi \in T_{p} M$ transverse to $H_{p} M$ and let $X=\psi_{*} \xi \in T_{P} S$. According to Proposition 2 we can find a family $G_{r}, r \in U \subseteq \boldsymbol{R}^{2 n}$, of maps $G_{r}: \bar{\Delta} \rightarrow \mathcal{C}$, analytic in $\Delta$, such that $G_{0}(1)=P,\left.(d / d s)\right|_{s=0} G_{0}\left(e^{i s}\right)=X$ and $G_{r}(\gamma), r \in \boldsymbol{R}^{2 n}$, smoothly foliates a neighborhood of $P$ in $S$.

Let

$$
f_{r}=\pi \circ G_{r}: \bar{\Delta} \rightarrow \boldsymbol{C}^{n+1} .
$$

Since $f_{r}(\gamma), r \in U \subseteq \boldsymbol{R}^{2 n}$, foliates a neighborhood of $p$ in $M$, for each point $q \in M$ (close to $p$ ) we can find a unique $r(q) \in \boldsymbol{R}^{2 n}$ and $\zeta_{q} \in \gamma \subseteq \partial \Delta$ such that $f_{r(q)}\left(\zeta_{q}\right)=q$. We set

$$
f^{q}(\zeta)=f_{r(q)}\left(\zeta_{q} \zeta\right), \quad \zeta \in \bar{\Delta} .
$$

It is clear that $f^{q}(1)=q$ and if $q^{\prime}=f^{q}\left(\zeta^{\prime}\right)$ for some $\zeta^{\prime} \in \partial \Delta$ then $f^{q^{\prime}}(\zeta)=f^{q}\left(\zeta^{\prime} \zeta\right)$. 
In this way we obtain a smooth family

$$
F: M \times \bar{\Delta} \rightarrow C^{n+1}, \quad F(q, \zeta)=f^{q}(\zeta),
$$

of immersed disks $f^{q}$ analytic in $\Delta$.

Clearly, $F_{s}(q)=f^{q}\left(e^{i s}\right)$ for small $s \in \boldsymbol{R}$ defines a local $\boldsymbol{R}$-action $\left(F_{s}\right)$ on $M$. Since $\left.(d / d s)\right|_{s=0} F_{S}(p)=\xi$, it is transverse to $H M$. We have to show that $\left(F_{S}\right)$ is a semi-extendable action. The holomorphicity of the mapping $G_{r}: \bar{\Delta} \rightarrow \mathcal{C}$ given by

$$
G_{r}(\zeta)=\left(f_{r}(\zeta),\left[H_{f_{r}(\zeta)} M\right]\right)
$$

for $\zeta \in \partial \Delta$ close to 1 plays a crucial role in this respect. We use first the holomorphicity of the second coordinate of $G_{r}$ to construct for each disc $f^{q}$ an $n$-dimensional complex subbundle $E_{q}$ of $\left(f^{q}\right)^{*} C^{n+1}$ as follows.

Set $f=f^{q}$ to simplify notation and recall that $f$ is the projection of a map

$$
G=(f, \tilde{f}): \bar{\Delta} \rightarrow \mathcal{C}=\boldsymbol{C}^{n+1} \times \boldsymbol{C} P^{n},
$$

which is holomorphic in $\Delta$. Using homogeneous coordinates for $\boldsymbol{C} P^{n}$, write

$$
\tilde{f}=\left[\tilde{f}_{1}: \tilde{f}_{2}: \ldots: \tilde{f}_{n+1}\right] .
$$

We have (see Proposition 3) that for $\zeta \in \gamma$,

$$
H_{f(\zeta)} M=\left\{X \in \boldsymbol{C}^{n+1} ; \tilde{f}(\zeta) \cdot X=0\right\} .
$$

Let $\sigma(z)$ be the 1 -form on $T_{f(z)}^{0,1} C^{n+1}$ given by

$$
\sigma(z)=\tilde{f}_{1}(z) d \overline{z_{1}}+\cdots+\tilde{f}_{n+1}(z) d \overline{z_{n+1}} .
$$

Then $\sigma$ is holomorphic for $z \in \Delta$ and nonvanishing at least for $z$ close to 1 . By pulling back with $f$, we get the 1 -form $f^{*} \sigma$ on $f^{*} T^{0,1} \boldsymbol{C}^{n+1}$.

For values of $z$ close to 1 let us denote by $E_{q}(z)$ the kernel of $f^{*} \sigma(z)$ in $f^{*} T^{0,1} \boldsymbol{C}^{n+1}$. Since we have the natural inclusion in $f^{*} T^{0,1} C^{n+1} \subset f^{*} T^{C} C^{n+1}$, we view $E(z)$ as an $n$ dimensional complex subbundle of $f^{*} T^{C} C^{n+1}$ that is holomorphic in $z \in \Delta$. Moreover, for $\zeta \in \gamma$ we have

$$
E_{q}(\zeta)=\left(f^{q}\right)^{*} H_{f^{q}(\zeta)}^{0,1} M
$$

To complete the proof we have to show that pull-back of the $H^{0,1} M$ bundle by the action $F_{s}$, given by

$$
\left[H_{q}^{0,1}(\zeta)\right]=F_{\zeta}^{*}(q)\left(\left[H_{F_{\zeta}(q)}^{0,1} M\right]\right)
$$

in a small neighborhood of 1 , has a holomorphic extension to the inside of $\Delta$. Instead of pulling back $H^{0,1} M$ by the action of $F_{S}$ we will construct a frame of holomorphic sections of $f^{*} T^{C} M$ and represent the section of $E_{q}(z)$ in this frame. This is achieved by pushing forward a fixed frame with the action $F_{S}$ as follows.

Pick a vector $Y \in T_{q}^{C} M, Y=Y_{1}+i Y_{2}$, where both $Y_{1}$ and $Y_{2}$ are tangent to $M$. Choose curves $\gamma_{i}:(-\varepsilon, \varepsilon) \rightarrow M$ such that $\gamma_{i}(0)=q, \dot{\gamma}_{i}(0)=Y_{i}(i=1,2)$. Let

$$
Y_{i}(\zeta)=\left.\frac{d}{d t}\right|_{t=0} f^{\gamma_{i}(t)}(\zeta)
$$


and $Y(\zeta)=Y_{1}(\zeta)+i Y_{2}(\zeta)$ for $\zeta \in \Delta$. It is clear by the definition that $Y(\zeta)$ is holomorphic in $\zeta \in \Delta$ and we can view it as a section of $\left(f^{q}\right)^{*} T^{C} C^{n+1}$. (Note that here the holomorphicity of the first component of $G_{r}$ was used in an essential way.)

Choose now a basis $\bar{Z}_{1}, \ldots, \bar{Z}_{n}$ of $H_{q}^{0,1} M$ and $X \in T_{q} M$ transverse to $H_{q} M$. Let $\bar{Z}_{i}(\zeta), Z_{i}(\zeta)$ and $X(\zeta)$ be the holomorphic sections obtained in the way described above.

Consider a frame of holomorphic sections $\eta_{k}(\zeta)$ of $E_{q}(\zeta)$ such that

$$
\left\langle\eta_{1}(1), \ldots, \eta_{n}(1)\right\rangle_{\boldsymbol{C}}=E_{q}(1)=\left(f^{q}\right)^{*} H_{q} M .
$$

For $\zeta \in \bar{\Delta}$ close to 1 the section $\eta_{k}(\zeta)$ is a linear combination of $\bar{Z}_{i}(\zeta), Z_{i}(\zeta)$ and $X(\zeta)$. We can write

$$
\eta_{k}(\zeta)=a_{k i}(\zeta) \bar{Z}_{i}(\zeta)+b_{k i}(\zeta) Z_{i}(\zeta)+c_{k}(\zeta) X(\zeta)
$$

where the coefficients $a_{k i}, b_{k i}, c_{k}$ are holomorphic in $\zeta$ for $\zeta \in \Delta$ close to 1 . This is equivalent to say that the pull-back of $\eta_{k}(\zeta)$ under the action $F_{\zeta}$ can be written:

$$
\left(F_{\zeta}\right)^{*} \eta_{k}(\zeta)=a_{k i}(\zeta) \bar{Z}_{i}+b_{k i}(\zeta) Z_{i}+c_{k}(\zeta) X \in T_{q}^{C} M
$$

for $\zeta=e^{i s}$ close to 1 . The action $\left(F_{s}\right)$ is semi-extendable if we show that the coefficients of the Beltrami tensor $\mu$ have a holomorphic extension. This follows from the holomorphicity of the coefficients $a_{k i}, b_{k i}, c_{k}$ in the same way as in the proof of Proposition 1.

By definition this amounts to showing that the circle bundle locally given by

$$
\left[H_{q}^{0,1}(\zeta)\right]=F_{\zeta}^{*}(q)\left(\left[H_{F_{\zeta}(q)}^{0,1} M\right]\right)
$$

has a holomorphic extension to the inside of $\Delta$ in a small neighborhood of 1 . To show this we use the holomorphicity of the mapping $G_{r}: \bar{\Delta} \rightarrow \mathcal{C}$ given by

$$
G_{r}(\zeta)=\left(f_{r}(\zeta),\left[H_{f_{r}(\zeta)} M\right]\right)
$$

for $\zeta \in \partial \Delta$ close to 1 . Since $M$ is an embedded hypersurface in $\boldsymbol{C}^{n+1}$, we have the canonical identification $H_{q} M \simeq H_{q}^{0,1} M$. Combining these two facts we conclude that

$$
E_{q}(\zeta)=\left[H_{F_{\zeta}(q)}^{0,1} M\right]
$$

has a holomorphic extension to the inside of $\Delta$ in a small neighborhood of 1 .

To show that $F_{\zeta}^{*}(q) E_{q}(\zeta)$ has a holomorphic extension we can work in local coordinates following the idea in the proof of Theorem 1.

Let us assume in the statement of Theorem 4 that the hypersurface $M$ is strongly pseudoconvex. Combining Theorems 3 and 4, we obtain the following

COROLLARY 1. A strongly pseudoconvex CR manifold of hypersurface type is locally embeddable if and only if admits a local semi-extendable action.

Moreover, under the assumption of strong pseudoconvexity we can even obtain a stronger conclusion than Theorem 4. Call an $\boldsymbol{R}$-action $\left(F_{s}\right)$ on $M$ contact if it preserves the contact bundle $H M$, i.e.,

$$
F_{s}^{*} H M=H M, \quad s \in \boldsymbol{R} .
$$

We then have 
Proposition 4. Let $M=M^{2 n+1}$ be an embedded hypersurface in $\boldsymbol{C}^{n+1}$, which is strongly pseudoconvex at the point $p \in M$. Then $M$ admits a (local) positive, semi-extendable contact $\boldsymbol{R}$-action in a neighborhood of $p$.

PROOF. The additional conclusion we have to prove here is the contact property of the action. This property does not seem to follow from the proof of Theorem 4. Instead of using the proof of Theorem 4 we sketch another argument which is based on a combination of results from [6], [17], [18].

Assume $M$ is a piece of a pseudoconvex hypersurface in $C^{n+1}$. By a suitable biholomorphic change of coordinates we may assume that locally $M$ is a piece of the boundary $\partial D$ of a strictly convex domain $D$ in $C^{n+1}$. Fix a point 0 in the interior of $D$. Let us denote by $B$ the unit ball in $C^{n+1}$. By the strict convexity of $D$ (cf. [17], [18]) there exists a mapping $F: B \rightarrow D, F(0)=0$, which is called a circular representation of $D$. It is shown in [19] for $n=1$ and in [6] in general that the circular representation of $D$ with base point 0 gives rise to an inner $S^{1}$-action on $\partial D$ in the sense of [6]. Using the circular representation $F$, we can push forward the standard circle action (induced by the Hopf fibration) from $B$ to $D$. By using the properties of the circular representation $F$ ([18], [6]), one easily checks that we obtain this way a semi-extendable and positive contact action on $M \subseteq \partial D$.

The converse of the above statement is contained in Theorem 3.

\section{REFERENCES}

[1] A. Andreotti and C. D. Hill, Complex characteristic coordinates and the tangential Cauchy-Riemann equations, Ann. Scuola Norm. Sup. Pisa 26 (1972), 299-324.

[2] K. Astala, Z. Balogh and H. M. Reimann, Lempert mappings and holomorphic motions in $\boldsymbol{C}^{n}$, Astérisque 261 (2000), 1-12.

[3] Z. M. Balogh and C. Leuenberger, Higher-dimensional Riemann maps, Internat. J. Math. 9 (1998), 421-442.

[4] Z. M. Balogh And C. Leuenberger, Quasiconformal contactomorphisms and polynomial hulls with convex fibers, Canad. J. Math. 51 (1999), 915-935.

[ 5 ] J. BLAND, Contact geometry and CR structures on $S^{3}$, Acta Math. 172 (1994), 1-49.

[ 6] J. Bland And T. Duchamp, Moduli for pointed convex domains, Invent. Math. 104 (1991), 61-112.

[7] A. Boggess, CR manifolds and the tangential Cauchy-Riemann complex, Stud. Adv. Math., CRC Press, Boca Raton, FL, 1991.

[8] M. S. Baouendi, P. Ebenfelt and L. Rothshild, Real submanifolds in complex space and their mappings, Princeton University Press, Princeton, NJ, 1999.

[9] M. S. BAouendi, L. P. RothsChiLd And F. TREVES, CR structures with group action and extendability of CR functions, Invent. Math. 82 (1985), 359-396.

[10] M. S. BAouendi And L. P. Rothschild, Transversal Lie group actions on abstract CR manifolds, Math. Ann. 287 (1990), 19-33.

[11] D. Catlin, Sufficient Conditions for the Extension of CR Structures, J. Geom. Anal. 4 (1994), $467-538$.

[12] E. M. CIRCA, Regularity of boundaries of analytic sets, Math. USSR Sb. 45 (1983), 291-336.

[13] C. D. Hill And C. M. NACINOviCH, Solvable Lie algebras and the embedding of CR manifolds, Boll. Unione Mat. Ital. B Artic. Ric. Mat. (8) 2 (1999), 121-126.

[14] H. Jacobowitz And F. TrEves, Non-realizable CR structures, Invent. Math. 66 (1982), 231-249. 
[15] A. Korányi And H. M. Reimann, Quasiconformal mappings on CR manifolds, Complex geometry and analysis (Pisa, 1988), 59-75, Lecture Notes in Math. 1422, Springer, Berlin, 1990.

[16] S. G. KRANTZ, Function theory of several complex variables, Second edition, Wadsworth-Brooks/Cole Math. Ser., Wadsworth \& Brooks/Cole Advanced Books \& Software, Pacific Grove, CA, 1992.

[17] L. Lempert, La métrique de Kobayashi et la représentation des domaines sur la boule, Bull. Soc. Math. France 109 (1981), 427-474.

[18] L. LEMPERT, Holomorphic invariants, normal forms, and the moduli space of convex domains, Ann. of Math. (2) 128 (1988), 43-78.

[19] L. LEMPERT, On three-dimensional Cauchy-Riemann manifolds, J. Amer. Math. Soc. 5 (1992), 923-969.

[20] C. Leuenberger, Lempert invariants and Riemann maps, Ph. D. thesis, University of Berne, 1997.

[21] A. MeZIANI, Perturbation of a class of CR structures of codimension larger than one, J. Funct. Anal. 116 (1993), 225-244.

[22] A. Newlander AND L. Nirenberg, Complex analytic coordinates in almost complex manifolds, Ann. of Math. 65 (1957), 391-404.

[23] L. NiRenberg, On a question of Hans Lewy, Russian Math. Surveys 29 (1974), 251-262.

[24] S. I. PINCHUK, A boundary uniqueness theorem for holomorphic functions of several complex variables, Mat. Zametki 15 (1974), 205-212.

[25] N. TANAKA, On the pseudoconformal geometry of hypersurfaces of the space of $n$ complex variables, J. Math. Soc. Japan 14 (1962), 397-429.

[26] F. Treves, Hypo-Analytic Structures: Local theory, Princeton Math. Ser. 40, Princeton University Press, Princeton, NJ, 1992.

[27] S. M. Webster, On the reflection principle in several complex variables, Proc. Amer. Math. Soc. 71 (1978), 26-29.

[28] S. M. Webster, On the proof of Kuranishi's embedding theorem, Ann. Inst. H. Poincaré Anal. Non Linéaire 6 (1989), 183-207.

UNIVERSITÄT BERN

MATHEMATISCHES INSTITUT

SIDLERSTRASSE 5

3012 BERN

SCHWEIZ

E-mail address: zoltan.balogh@math-stat.unibe.ch
ECOLE D'INGÉNIEURS ET

D'ARCHITECTES DE FRIBOURG

BD. DE PÉROLles 80

1705 FRIBOURG

SUISSE

E-mail address: christoph.leuenberger@eif.ch 